\title{
In Vivo Vector Flow Imaging Using Improved Directional Beamforming
}

Henze, Lasse; Holfort, Iben Kraglund; Kortbek, Jacob; Hansen, Kristoffer Lindskov; Jensen, Jørgen Arendt

\section{Published in:}

Proceedings of IEEE Ultrasonics Symposium

Link to article, DOI:

10.1109/ULTSYM.2007.613

Publication date:

2007

Document Version

Publisher's PDF, also known as Version of record

Link back to DTU Orbit

Citation (APA):

Henze, L., Holfort, I. K., Kortbek, J., Hansen, K. L., \& Jensen, J. A. (2007). In Vivo Vector Flow Imaging Using Improved Directional Beamforming. In Proceedings of IEEE Ultrasonics Symposium (pp. 2438-2441). IEEE. https://doi.org/10.1109/ULTSYM.2007.613

\section{General rights}

Copyright and moral rights for the publications made accessible in the public portal are retained by the authors and/or other copyright owners and it is a condition of accessing publications that users recognise and abide by the legal requirements associated with these rights.

- Users may download and print one copy of any publication from the public portal for the purpose of private study or research.

- You may not further distribute the material or use it for any profit-making activity or commercial gain

- You may freely distribute the URL identifying the publication in the public portal 


\title{
In Vivo Vector Flow Imaging Using Improved Directional Beamforming
}

\author{
Lasse Henze $^{1}$, Iben Kraglund Holfort ${ }^{1}$, Jacob Kortbek ${ }^{12}$, Kristoffer Lindskov Hansen ${ }^{3}$ and Jørgen Arendt \\ Jensen $^{1}$
}

1) Center for Fast Ultrasound Imaging, Ørsted•DTU, Bldg. 348,

Technical University of Denmark, DK-2800 Kgs. Lyngby, Denmark

2) B-K Medical, Mileparken 34, 2730 Herlev, Denmark

3) University hospital of Copenhagen, Rigshospitalet Blegdamsvej 9, DK-2100 Copenhagen

\begin{abstract}
Directional beamforming has shown promising results for creating vector flow images. The method measures both the flow angle and the magnitude of the velocity. The flow angle is estimated by focusing lines in a range of angles from 0 to 180 degrees. The true angle is identified as the angle that produces the largest correlation coefficient across emissions. The magnitude of the velocity is found by cross-correlating consecutive lines focused along the direction of flow, to find the spatial shift corresponding to the velocity. In initial in vivo experiments, the method has however shown weaknesses by yielding outliers when a substantial clutter signal is present after clutter filtering. This is especially a problem when the flow angle is close to 90 degrees as the slow time frequencies from the flow signal is similar to that of the clutter, making clutter filtration difficult. When the angle determination fails, the correct velocity can no longer be found. The purpose of this work is to improve the robustness of the directional beamforming method, making precise in vivo measurement possible. A more robust angle estimator is proposed. Spatial averaging in the axial direction is applied over a depth of 2 wavelengths. Instead of traditionally beamforming a single line, three lines are beamformed with an interline distance of a wavelength. To improve clutter filtering a post correlation clutter filter is applied, by removing peaks in the correlation functions corresponding to low velocities, since these peaks are believed not to be a result of the measured flow. The method has been tested on a flow phantom, using the RASMUS experimental scanner. The flow had a parabolic velocity distribution with a peak velocity of $0.1 \mathrm{~m} / \mathrm{s}$, and a flow angle of 90 degrees. The measurement were made with a $6.2 \mathrm{MHz}$ linear array transducer, using 30 emissions and 128 transducer elements for each estimate. Using the same measurement setup, an initial in vivo study has been carried out. The measurements have been performed on the carotid artery of 11 human volunteers. To validate the method MR angiography has been performed on all human volunteers as a gold standard. For the phantom measurement $76.30 \%$ of the angle estimates are within +-5 degrees of the actual angle, when using the traditional setup. Using our new approach $98.32 \%$ of the angle estimates are within +-5 degrees from the true angle. The comparison between the stroke volume measurements in the carotid artery calculated using directional beamforming and MR angiography, gives a correlation coeficient of 0.84 . Phantom and in vivo measurements has been carried out with a more robust implementation of the directional beamforming method. With the applied changes, the method has shown improved results for in vivo measurements.
\end{abstract}

\section{INTRODUCTION}

In traditional ultrasonic velocity estimation systems, only the axial velocity component is estimated. So to find the absolute velocity, the beam to flow angle must be found. The absolute velocity is an important clinical parameter, so the ability to measure it accurately is of great value. Jensen [1] suggested to cross-correlate lines beamformed in the flow direction, to directly measure the magnitude of the velocity vector. The method performs well, even for pure transverse flow, but needs a priori knowledge of the flow angle. To find the flow angle Kortbek and Jensen [2] suggested to beamform lines in a range of directions, from 0 to 180 degrees, and then search for the direction with the largest correlation over time. It was shown in simulations and phantom measurements that the method was capable of finding both the correct flow angle and velocity magnitude using few emissions. However when Holfort [3] performed initial in vivo experiment is was shown that the angle estimator breaks down when a substantial amount of clutter is present after clutter filtering. When the angle determination fails, the correct velocity can no longer be found. The purpose of this work is to improve the robustness of the angle estimator to enable accurate velocity vector estimation. This is performed by applying spatial averaging, and improving clutter filtering.

\section{METHODS AND MATERIALS}

\section{A. Angle Estimation}

To find the flow angle, directional signals are beamformed in a range from 0 to 180 degrees as shown in Fig 1. The directional signal is given by $S_{i}\left(x_{\phi}^{\prime}\right)$, where $i$ is the emission number $\phi$ is the beam angle, as seen on the figure and $x^{\prime}$ is the spatial location on the line

The directional signal, corresponding to the true flow angle, for emission $i$, contains the same blood scatterers, as for emission $i+1$, excepts for the few scatterers that has left the signal in the end or entered at the start. This will make the correlation between consecetive signals high. For the remaining angles, a different distribution of scatters will be examined for each emission, due to the misalignment between the signals and the direction of flow. As a result the correlation will be low. The directional signal corresponding to the true flow angle, can therefore be identified as the signal that produces the largest normalized correlation coefficient over emissions. The correlation coefficient for different angles is calculated using 


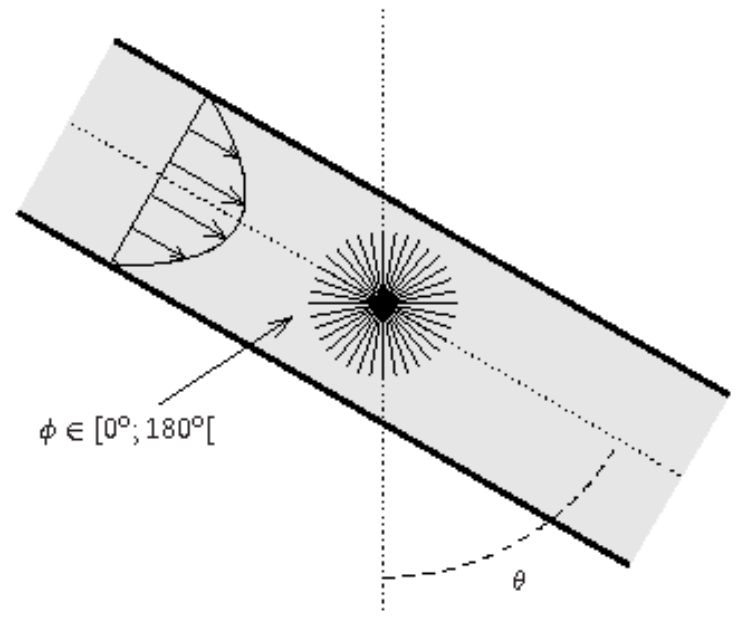

Fig. 1. Setup for the method. Directional signals are beamformed from 0 to 180 degrees at the estimation point. The directional signals are correlated over emissions. The signal with the largest correlation is identified as the direction of flow. When the direction of flow is found, the magnitude of the velocity is found by cross-corelating consecutive signals to find the spatial shift corresponding to the blood velocity.

$$
P(\phi)=\max \left\{\sum_{i=1}^{N-1} C\left(S_{i}\left(x_{\phi}^{\prime}\right), S_{i+1}\left(x_{\phi}^{\prime}\right)\right)\right\},
$$

where $\mathrm{N}$ is the total number of emissions and $\mathrm{C}$ is the normalized correlation coefficient defined by

$$
C(a, b)=\frac{R_{a b}(k)-\mu_{a} \mu_{b}}{\sqrt{\left[R_{a a}(0)-\mu_{a}^{2}\right]\left[R_{b b}(0)-\mu_{b}^{2}\right]}},
$$

where $R_{a b}$ is the cross-correlation between signal $a$ and $b$, $\mu_{a}$ is the mean of $a$ and $\mu_{b}$ is mean of $b$. The sum in (1) is introduced to perform averaging.

The angle can now be estimated by searching for the largest correlation coefficient,

$$
\theta_{e}=(\underset{\phi}{\arg \max }\{P(\phi)\})
$$

\section{B. Velocity Estimation}

When the direction of flow is known, the magnitude of the velocity can be found. Two signals taken from consecutive emissions, beamformed along the direction of flow, is related to each other by a spatial shift $x_{s}^{\prime}$ given by

$$
S_{i}\left(x_{\phi}^{\prime}\right)=S_{i+1}\left(x_{\phi}^{\prime}-2 V T_{p r f}\right)
$$

where $V$ is magnitude of the velocity and $T_{p r f}$ is the time between emission. The spatial shift is found be locating the peak of the cross-correlation function taken between two consecutive signals.

$$
x_{s}^{\prime}=\underset{k}{\arg \max } \sum_{i=1}^{N-1} R_{S_{i} S_{i+1}}(k),
$$

The velocity is now given by

$$
V=\frac{x_{s}^{\prime}}{T_{p r f}}
$$

For a more in depth description of the basic method see [2]

\section{Spatial Averaging}

To improve the robustness, spatial averaging is applied on the angle estimator in the axial dimension. The spatial averaging is applied by beamforming three lines for every angle. The three lines are beamformed with in a range of $2 \lambda$. The new angle estimator is give by

$$
P(\theta)=\max \left\{\sum_{j_{z}=1}^{3} \sum_{i=1}^{N-1} C\left(S_{i, \theta,\left(j_{z}-2\right) \lambda}(t), S_{i+1, \theta,\left(j_{z}-2\right) \lambda}(t)\right)\right\},
$$

The length of the directional signals, in the used setup, is $20 \lambda$, so the applied spatial averaging will only marginally reduce the spatial resolution of the estimator.

\section{Post Correlation Clutter Filtering}

To improve clutter filtering, peaks in the correlation function taken in (2) identified to be a product of the clutter is removed. The Directional signals are composed of two major components, one flow component, coming from the blood scatterers and one clutter component, coming from the stationary tissue,

$$
S_{i}\left(x^{\prime}\right)=F_{i}\left(x^{\prime}\right)+N\left(x^{\prime}\right),
$$

where $F_{i}\left(x^{\prime}\right)$ is the flow component and $N\left(x^{\prime}\right)$ is the clutter component. It is assumed that the clutter and blood component is uncorrelated and that the clutter component is stationary over emissions. When the cross-correlation is taken in (2) between two consecutive emissions, it corresponds to the cross-correlation of the flow signal, added with the autocorrelation of the clutter signal

$$
R_{S_{i}\left(x^{\prime}\right), S_{i+1}\left(x^{\prime}\right)}=R_{F_{i}\left(x^{\prime}\right), F_{i+1}\left(x^{\prime}\right)}+R_{N\left(x^{\prime}\right), N\left(x^{\prime}\right)}
$$

It is known from basic signal processing, that the autocorrelation function peaks at lag zero and from (4) it is known that the cross-correlation of the flow signal, for the true angle, peaks at lag $2 V \frac{T_{p r f}}{c}$. So the post correlation clutter filtering is done by ignoring peaks laying around lag zero in (2).

By doing this, peaks emerging as a result of the clutter is removed, without affecting the true peak coming as a result of the measured flow.

\section{E. Measurements}

To test the performance of the method, phantom and in-vivo measurements have been carried out on the experimental ultrasound scanner RASMUS [4]. The parameters used for all measurements can be seen in Table I. 
Phantom measurements have been performed on a circulating flow rig as seen on Fig 2. A long tube is connected to a flow pump. The transducer is placed in a fixture, with a known beam to flow angle. An electro magnetic flow meter is placed around the tube to read the flow, as a reference. Measurements has been carried out for a beam to flow angle of 90 degrees.

An transmit focus is placed in double the depth of the tube, to get a wide transmit beam. The vector flow images has been created by measuring 16 flow lines pr image, with an axial spacing of $\lambda$ between each estimate. 64 elements is used in transmit and 128 elements is used in receive. The used scanner has access to 128 channels, so a sliding aperture can only be achieved in transmit. To avoid tilting of the point spread function, a boxcar apodization is applied in receive to force a symmetric receive aperture.

The in-vivo measurements have been carried out on the carotid artery of 11 healthy human volunteers. To validate the method MR angiography has been performed on all 11 volunteers as an gold standard. To enable a comparison, the volume flow over a heart cycle has been calculated. The volume flow has been calculated using the assumption of rotational symmetry. All in-vivo measurements has been performed by an experienced medical doctor.

Prior to the in-vivo measurements, the intensities of the transmit setup have been measured using a hydrophone. All measured intensities where below the limits set by the U.S Food an Drug Administration [5].

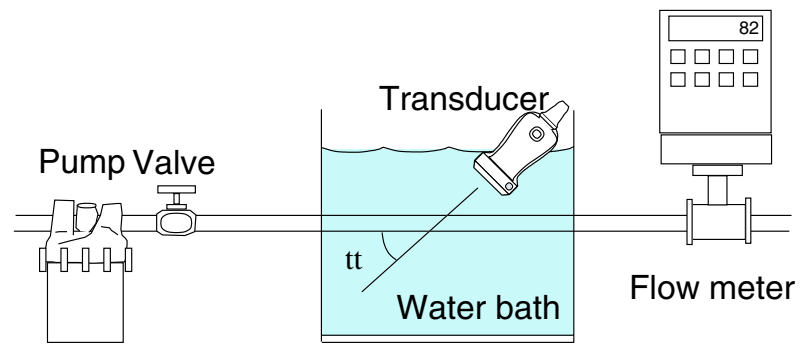

Fig. 2. Setup of the used flowrig. A pump secures a steady parabolic flow in the tube. A fixture holds the transducer at a known beam to flow angle. An electro magnetic flow meter measures the volume flow as a reference.

\section{EXPERIMENTAL RESULTS}

\section{A. Phantom Measurements}

The top graph in Fig. 3 shows the angle estimates, for the phantom measurement, calculated using the traditional setup. Angle estimates from 10 images each containing 16 flow lines are plotted. $76.3 \%$ of the estimates are within $\pm 5 \%$ from the correct angle. The angle estimates, at the side of the tube, are slightly worse than the estimates in the middle. The method performs best on the central flow lines. This is because they have the largest recieve aperture.

The bottom of Fig. 3 shows angle estimates, calculated with the improvements. $98.32 \%$ of the estimates are with in \pm
TABLE I

STANDARD PARAMETERS FOR PHANTOM AND IN-VIVO MEASUREMENTS

\begin{tabular}{lcc}
\hline Type & & Linear array \\
Transducer center frequency & $f_{0}$ & $7 \mathrm{MHz}$ \\
Wavelength & $\lambda$ & $0.22 \mathrm{~mm}$ \\
Pitch of transducer elements & $w$ & $0.208 \mathrm{~mm}$ \\
Height of transducer elements & $h_{e}$ & $4.5 \mathrm{~mm}$ \\
Kerf & $\mathrm{ke}$ & $0.035 \mathrm{~mm}$ \\
Number of active elements(receive/transmit) & $N_{e}$ & $128 / 64$ \\
Apodization(receive/transmit) & & Boxcar/Hanning \\
Number of excitation periods & & 2 \\
Elevation focus & $R_{e}$ & $25 \mathrm{~mm}$ \\
\hline Radius of tube & $R$ & $6 \mathrm{~mm}$ \\
Peak velocity in flow profile & $v_{0}$ & $0.15 \mathrm{~m} / \mathrm{s}$ \\
Beam to flow angles & $\theta$ & $90^{\circ}$ \\
\hline RF lines for estimation & $N$ & 32 \\
Length of directional signals & & $20 \lambda$ \\
Sampling frequency & $f_{s}$ & $40 \mathrm{MHz}$ \\
Number of oscillations & $N_{o}$ & 2 \\
Speed of sound & $c$ & $1480 / 1540 \mathrm{~m} / \mathrm{s}$
\end{tabular}

$5 \%$ from the correct angle. A slight worsenning of the estimates can again be observed at the side of the tube, but not to the same extend as in the top figure. A fairly even performance is observed over the individual flow lines. Using the improvements a satisfactory result is optained for the phantom measurements.

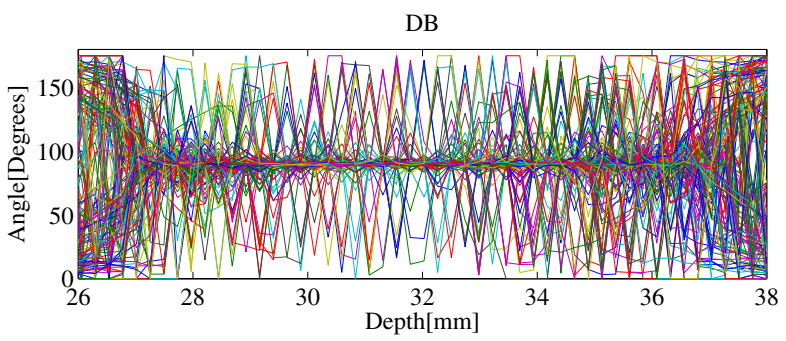

Improved DB

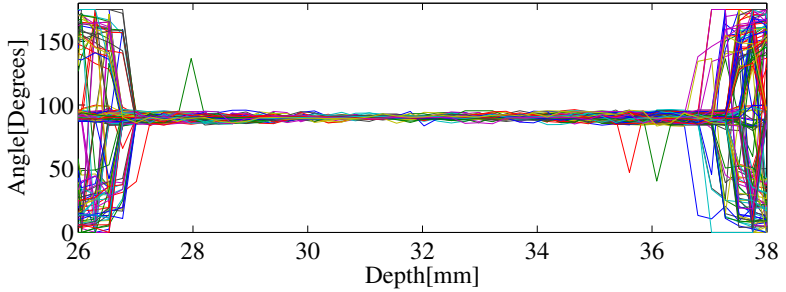

Fig. 3. Angle estimates from the phantom measurements, for a beam to flow angle of 90 degrees. The top figure shows the estimates calculated with a direct implementation of Directional beamforming. The bottom figure shows the estimates calculated with the improved version of Directional beamforming.

\section{B. In-vivo Measurements}

The top of Fig 4 shows a velocity vector image of the common carotid artery and jugular vein, calculated using the traditional setup. The brightness of the color and the length of the arrows, shows the magnitude of the velocity and the direction of the arrows shows the flow angle. The main direction of flow in the artery and vein can be observed, but the image is dominated by errors. 
The bottom of Fig 4 shows, for the same frame as the top of the figure, a velocity vector image, calculated using the new method. A more believable result is obtained and only few estimates can visually be determined to be errors.

Fig 5 shows the regression line for stroke volume, estimated using Directional beamforming and MR angiography. The MR measurements directly measures the volume flow, where as the ultrasound measurements is based on the assumption of a rotational symmetric velocity field. The vertical line on each of the data points, show the possible distribution of volume flow estimates, if the MR measurements where based on the same assumptions as the ultrasound measurements. The correlation coefficient is calculated to be 0.84 , with a $95 \%$ confidence interval from 0.49 to 0.96 .

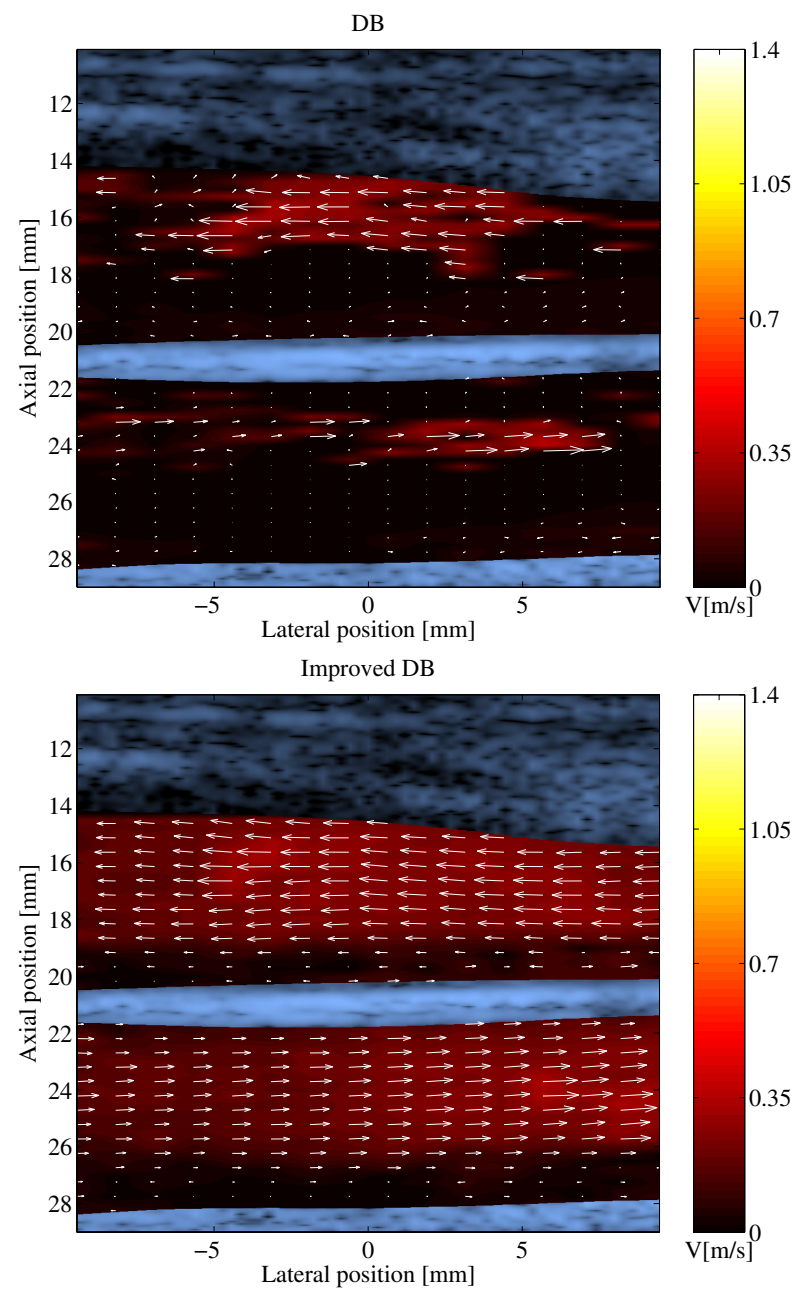

Fig. 4. In vivo scan of the carotid artery and jugular vein. The top figure shows the estimates calculated with a direct implementation of Direction beamforming. The bottom figure shows the estimates calculated with the improved version of Direction beamforming.

\section{CONCLUSION}

A more robust version of Directional beamforming has been implemented. The method has been tested in circulating flowrig. 11 successful in vivo measurements have been carried out. MR angiography has been performed as a reference. A

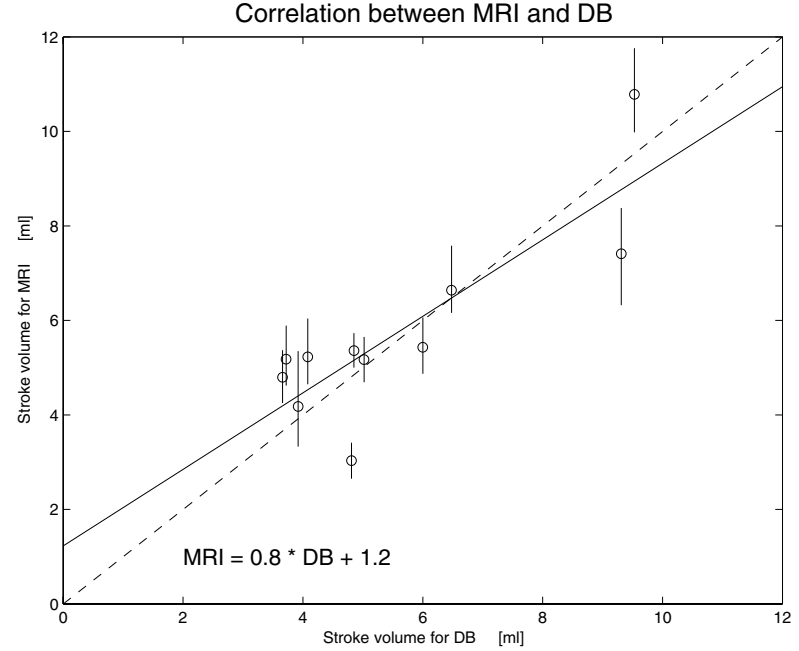

Fig. 5. Regression line for volume flow estimated with MR and ultrasound. The vertical line on each of the data points shows the uncertainty of the ultrasound estimates, due to the assumption of rotational symmetry.

corelation coefficient of 0.84 has been achieved for stroke volume estimated using MR and ultrasound. The preliminary results look encouraging for further research.

\section{ACKNOWLEDGMENT}

This work was supported by the Faculty of Health Science, University of Copenhagen, by grant 274-05-0327 from the Danish Research Agency, the Radio-parts foundation and by B-K Medical A/S, Denmark.

\section{REFERENCES}

[1] J. A. Jensen, "Directional velocity estimation using focusing along the flow direction: I: Theory and simulation," IEEE Trans. Ultrason., Ferroelec., Freq. Contr., pp. 857-872, 2003.

[2] J. Kortbek and J. A. Jensen, "Estimation of velocity vector angles using the directional cross-correlation method," IEEE Trans. Ultrason., Ferroelec., Freq. Contr., vol. 53, pp. 2036-2049, 2006.

[3] I. K. Holfort, J. Kortbek, and J. A. Jensen, "Vector velocity imaging using cross-correlation and virtual sources," in Proc. IEEE Ultrason. Symp., 2006, pp. 2023-2026.

[4] J. A. Jensen, O. Holm, L. J. Jensen, H. Bendsen, S. I. Nikolov, B. G. Tomov, P. Munk, M. Hansen, K. Salomonsen, J. Hansen, K. Gormsen, H. M. Pedersen, and K. L. Gammelmark, "Ultrasound research scanner for real-time synthetic aperture image acquisition," IEEE Trans. Ultrason., Ferroelec., Freq. Contr., vol. 52 (5), pp. 881-891, May 2005.

[5] FDA, "Information for manufacturers seeking marketing clearance of diagnostic ultrasound systems and transducers," Tech. Rep., Center for Devices and Radiological Health, United States Food and Drug Administration, 1997. 